\title{
Correcting physiological and biochemical status of service dogs with dihydroquercetin
}

\author{
Galina Molyanova ${ }^{1, *}$, Vladimir Ermakov ${ }^{1}$, Irina Bistrova ${ }^{1}$ \\ ${ }^{1}$ Samara State Agricultural Academy, st.Uchebnaya, 2, Kinel, Russia, 446442
}

\begin{abstract}
The search for new ways to optimize the adaptive capabilities of service dogs is a prerequisite for increasing the viability of animals and increasing their longevity. Dihydroquercetin neutralizes the increased level of free radicals toxic to the body, protecting cell membranes from destruction, and reduces the harmful effects of oxidative stress. The use of dihydroquercetin contributed to increased providing cells with oxygen, stimulated anabolic processes and increased the antioxidant defense of the body. The amount of red blood cells in the blood of service dogs increased by $11.5-19.1 \% \quad(p<0.01)$ when using dihydroquercetin, hemoglobin increased by $5.7-9.4 \%(\mathrm{p}<0.05)$, total protein by $21-32 \%(\mathrm{p}<0.01)$, albumin by $30-31 \%(p<0.001)$, compared with similar data at the beginning of the experiment. The antioxidant properties of dihydroquercetin contributed to the reduction of malondialdehyde by $23.9 \%(\mathrm{p}<0.05)$; reduction of diene conjugates by $26 \%$; and an increase in catalase activity by $36 \%$ compared to the data on the first day of the experiment. The use of dihydroquercetin optimizes the adaptive capabilities of service dogs and can be used to increase the vitality and longevity of animals.
\end{abstract}

\section{Introduction}

Service dog breeding is the oldest livestock industry and plays an important role in the modern world. One of the requirements for dogs in cynology is high service (working) and breeding abilities. These animal qualities are closely interrelated with the physiological status of the body, which is significantly influenced by the conditions of detention, nutrition, treatment of dogs and many other factors. The emergence of secondary immunodeficiencies and metabolic disorders in dogs is also facilitated by following stress factors: the placement of kennels in cities, urbanization, increased gas contamination and dustiness of air, prolonged transportations. Active work of the dog's muscles leads to increased consumption of energy and nutrients in the body, such as protein, vitamins, minerals, fats and carbohydrates. As a result, the indicators of the body's immune system decrease with the combined effect of abovementioned factors, which can lead to an increased susceptibility of animals to diseases of various etiologies and poor performance.

The average tour of duty for service dogs is 5-6 years, one year of which is spent on training and raising a dog. Dogs begin training no earlier than 8 months of age, that is, total service life is 2-4 years on average. The search for new ways to optimize the adaptive

* Corresponding author: Molyanova@yandex.ru 
capabilities of service dogs is a prerequisite for increasing the viability of animals and their longevity.

The wide biological activity amplitude of flavonoids is associated with the diversity of their chemical structures and the various physical and chemical properties resulting from them. Flavonoids have antioxidant, capillary-protective, choleretic, hepatoprotective, cardioprotective, anti-atherosclerotic, anti-inflammatory, antimicrobial, antiviral and other types of pharmacological properties [1-7].

Dihydroquercetin is a natural flavonoid isolated from larch wood. It has a wide range of biological and vitamin activity and serves as a source of vitamin $\mathrm{P}$, is recognized as a perfect antioxidant and is widely used in medicine and the food industry [8-11].

Scientists Vladimirov Yu.A., Proskurina E.V. and others studied the problem of regulating apoptosis using antioxidants. Studies have shown that the peroxidase activity of the cytochrome with dioleyl cardiolipin complex is decreased by $50 \%$ in the presence of dihydroquercetin and other antioxidants. Thus, flavonoids can have an inhibitory effect on the development of apoptosis in its three units associated with the formation of free radicals [12].

The balance between the oxidation of fatty acids and their reduction is determined by the formation ratio of reactive oxygen intermediates and the functional state of antioxidant systems, including superoxide dismutase, peroxidase, catalase and bioantioxidants, which are donors of sulfhydryl groups, tocopherols, catechins, ascorbic acid, etc. There is a sharp decrease in the antioxidant activity of tissues and an equilibrium shift towards lipid oxidation during hypoxia. Flavonoids, having the ability to inhibit the peroxidation of lipids that make up the membranes of cells and organelles, thereby stabilize the membranes, which is considered the leading mechanism for protecting the body from various damaging effects, including hypoxia, with antioxidants. Researchers have proven that an early marker of antioxidant defenses is activation of superoxide dismutase with moderate activity of chronic hepatitis. In the advanced stages of the disease and with high activity of the pathological process, there is a tendency to a decrease in the activity of antioxidant enzymes, especially catalase [13-19].

The dihydroquercetin deserves attention among the substances with antimicrobial action. The possibility of its use in animal husbandry was studied by such researchers as Artemiev O.A. and Pereselkova D.A. They evaluated the effect of different concentrations of standard antibiotics and dihydroquercetin solutions on pathogenic and conditionally pathogenic microorganisms under invitro conditions by using agar diffusion methods. Staphylococcus Epidermidis appeared to be highly sensitive to a $5.0 \%$ solution of dihydroquercetin and showed a diameter of the inhibition zone (IZ) of $21.33 \pm 0.82 \mathrm{~mm}$. The probiotic Escherichia Coli and non-pathogenic microflora representatives of the gastrointestinal tract Micrococcus Luteus (lysodeicticus) ATCC 4698 and Micrococcus Luteus ATCC 10240 were insensitive to dihydroquercetin concentrations from 0.5 to $2.0 \%$ [20].

Krutikova E.V. and Fomichev Yu.P. revealed a positive effect on protein, nitrogen, lipid and mineral metabolism in the course of scientific and industrial experience, when a natural antioxidant dihydroquercetin was added to the diet. The use of dihydroquercetin improved the functional state of the liver, as well as antioxidant defense of the body; contributed to an increase in the supply of oxygen to the body's cells. As a result, the intensity and anabolic orientation of the metabolism improved [21].

Researchers Tseluyko S.S., Gorbunov M.M. and others found that the use of dihydroquercetin allows saving the structure of the airways under low temperatures and reduce lipid peroxidation. The combined use of antioxidants enhances regeneration in the airways, normalizes the intensity of lipid peroxidation and the level of antioxidant defense [22]. 
Kharchenko Yu.A. revealed during the course of scientific and industrial experiment that giving the bioflavonoid complex to dairy piglets as a feed additive at a dose of $1.0 \mathrm{~g} / \mathrm{kg}$ of body weight helps to increase average daily growths (by 19.1\%), reduce the physiological values of alanine aminotransferase activity (by $14.5 \%$ ) and alkaline phosphatase (by $6.2 \%$ ). The phagocytic activity of neutrophils increases (by $24.2 \%$ ), the amount of albumin in the serum increases (by 34.7\%), as well as the content of immunoglobulins in the serum (by 13.5\%) [23].

It was established by the researcher Kolesnikov A.V. that adaptation indicators for the formation of nonspecific protective forces in the body of calves receiving dihydroquercetin were higher than in control animals. Leukocyte phagocytic activity was higher by 12.42 ; bactericidal activity of blood serum by $6.47 \%$; lysozyme by $4.43 \%$. At 180 days of age, the live weight of calves in the experimental animal group was $145.23 \pm 2.51 \mathrm{~kg}$, which is $3.4 \%$ higher than the control data; the average daily increase was $8.0 \%(\mathrm{p} \leq 0.01)$ [24].

Scientists Yeskov E.K. and Tinaev N.I. added dihydroquercetin in the drinking water for young rabbits. They found a positive effect of microdoses of this drug on live weight (an increase of 6.9\%) and feed conversion (a decrease of 13.9\%). The economic effect per 1 $\mathrm{kg}$ of slaughter weight was 11.92 rubles [25].

Based on the foregoing, the correction of the physiological and biochemical status of service dogs with the dihydroquercetin antioxidant will be economically feasible for cynology.

\section{Materials and methods}

Scientific experiment was carried out in the areal center conditions of the Dog Training Service of Main Directorate of the Russian Ministry of Interior in the Samara Region. The German shepherd dogs were clinically healthy and aged 2-4 years with an average live weight of $30 \mathrm{~kg}$ against the conditions of keeping and feeding accepted at the enterprise. Groups of animals were formed according to the pair-analogue principle with 10 individuals in each. Dogs in group 1 (experimental) received the main diet and dihydroquercetin at a dose of $0.01 \mathrm{~g} / \mathrm{kg}$ live weight in a capsule 1 time per day with meals. 2 group (control) had the basic diet. Hematological and biochemical blood tests were performed on the basis of the GNU Samara NIVS. Blood sampling for analysis was carried out from the superficial vein of the forearm before feeding in the morning hours at the beginning of the experiment on the 10th, 20th and 40th day from the start of using the drug. The data obtained during the experiment were processed using biometrics with the calculation of generally accepted constants and using the STADIA software.

\section{Research results}

During the experiment, the physiological state of animals in the control and experimental groups did not go beyond the physiological norms.

The dihydroquercetin effect on the animal organism manifested itself in a quantitative change of the blood corpuscles, the concentration of hemoglobin in red blood cells comparing to the data of the indicators at the beginning of the experiment.

The number of red blood cells on the 1 st day of the experiment was $5.9 \pm 0.25 \cdot 10^{12} / 1$. On day 20 , the number of red blood cells was $6.5 \pm 0.21 \cdot 10^{12} / 1$, which is higher by $11.5 \%$ $(\mathrm{p}<0.05)$, on day 30 the amount was $19.1 \%(\mathrm{p}<0.01)$ higher than at the beginning of experiment. Also, a hemoglobin increase in the blood of animals on the 20th and 30th day of the experiment was detected, by $5.7 \%(\mathrm{p}<0.05)$ and $9.4 \%(\mathrm{p}<0.05)$, respectively. The 
shepherd dogs treated with dihydroquercetin in addition to the basic diet had the best hematological data.

In the body, the crude protein has the following functions: participates in blood coagulation, maintains a constant blood $\mathrm{pH}$, performs a transport function, participates in immune reactions and many other. The amount of crude protein increased on the 20th day of antioxidant intake by $21 \%(\mathrm{p}<0.001)$, on the 30 th day by $32 \%(\mathrm{p}<0.001)$ compared to the data at the beginning of the experiment. The content of albumin protein fraction was higher than globulin. The amount of albumin in the blood plasma of dogs on the 20th and 30th day of the experiment was higher by $30-31 \%(\mathrm{p}<0.001)$ compared with the initial data, which indicates a more intense level of anabolic processes in the animal body.

The content of bilirubin in the blood is most often determined from tests of the functional state of the liver in order to assess pigment function. Aspartate aminotransferase (AST) and alanine aminotransferase (ALT) are determined to assess enzyme function.

Table 1. Biochemical blood parameters of dogs treated with dihydroquercetin.

\begin{tabular}{|c|c|c|c|c|}
\hline \multirow{2}{*}{ Indicator } & \multicolumn{4}{|c|}{ Day of experiment } \\
\cline { 2 - 5 } & Day 1 & Day 10 & Day 20 & Day 30 \\
\hline Crude protein, g/l & $50.9 \pm 1.71$ & $59.3 \pm 1.53^{*}$ & $63.6 \pm 1.49^{* *}$ & $67.6 \pm 1.41^{* *}$ \\
\hline Albumin, g/1 & $28.4 \pm 1.41$ & $38.4 \pm 1.41^{*}$ & $38.1 \pm 1.47^{* *}$ & $37.4 \pm 1.32^{* *}$ \\
\hline Total bilirubin, $\mu \mathrm{mol} / 1$ & $3.1 \pm 0.15$ & $2.5 \pm 0.13$ & $1.63 \pm 0.26^{* *}$ & $1.54 \pm 0.08^{*}$ \\
\hline AST, IU/1 & $31.2 \pm 1.21$ & $30.4 \pm 1.31$ & $28.6 \pm 1.09$ & $28.2 \pm 1.52^{*}$ \\
\hline ALT, IU/1 & $23.6 \pm 1.14$ & $23.93 \pm 0.95$ & $25.8 \pm 1.07$ & $24.2 \pm 1.5$ \\
\hline ASAT/ALAT ratio & 1.32 & 1.27 & 1.11 & 1.16 \\
\hline Cholesterol, mmol/1 & $3.46 \pm 0.24$ & $3.73 \pm 0.20$ & $3.78 \pm 0.34$ & $4.68 \pm 0.28^{* *}$ \\
\hline Glucose, mmol/1 & $6.24 \pm 0.37$ & $5.21 \pm 0.27$ & $5.06 \pm 0.33$ & $5.02 \pm 0.42$ \\
\hline \multicolumn{5}{|c|}{ Hereinafter, a note: ${ }^{*}-\mathrm{p} \leq 0.05, * *-\mathrm{p} \leq 0.01-$ compared to day 1 data } \\
\hline
\end{tabular}

AST contributes to the formation of oxaloacetic acid from the amino acid aspartate. The intensity of the transamination process depends on the activity of the enzyme. ALT acts as a catalyst for the reversible transfer of alanine from the amino acid to alpha-ketoglutarate. As a result of the amino group transfer, glutamic and pyruvic acids are obtained. Alanine is an amino acid that can quickly turn into glucose. Due to this, energy for the work of the brain and central nervous system is obtained. The AST enzyme activity in the blood plasma of dogs treated with dihydroquercetin was lower by $8.3 \%$ on the 20 th day, by $16 \%$ on the 30 th day; ALT is higher by $2.5-9 \%$, respectively, compared with the data at the beginning of the experiment. The dynamics of the De Ritis ratio indicates the predominance of anabolic processes in the bodies of dogs.

In order to assess cholesterol- and glucose-forming functions, the blood levels of cholesterol and glucose are determined. Cholesterol levels indicate the amount of substrate for building cell membranes and providing an endogenous metabolic response to stress. The cholesterol content in the blood serum of shepherd dogs on the 10th and 20th day of the experiment was higher by 8.1 and $9.0 \%$, respectively, compared to the data on the first day, and by $32.5 \%$ on the 30 th day of the experiment, which indicates a positive dihydroquercetin effect on the cholesterol-forming function of the liver.

The main indicator of carbohydrate metabolism is blood glucose. Glucose sustains the normal functioning of individual cells, organs and the body as a whole. The blood glucose in dogs was within normal physiological limits and was at the level of 4.3-7.3 $\mathrm{mmol} / \mathrm{l}$, while its content at the beginning of the experiment was $6.24 \mathrm{mmol} / \mathrm{l}$, which is close to the upper limit of physiological norm and may indicate an increased function of the adrenal cortex as a response to stress.

\begin{tabular}{|l|c|c|c|c|}
\hline \multicolumn{4}{|c|}{ Table 2. The dynamics of the dog antioxidant system after dihydroquercetin treatment. } \\
\hline & Day of experiment \\
\cline { 2 - 5 } & control & Day 10 & Day 20 & Day 30 \\
\hline
\end{tabular}




\begin{tabular}{|c|c|c|c|c|}
\hline Malondialdehyde, $\boldsymbol{\mu m o l} / \mathbf{l}$ & $22.1 \pm 1.1$ & $20.8 \pm 0.49$ & $19.9 \pm 0.91^{*}$ & $16.8 \pm 0.87^{* *}$ \\
\hline Diene conjugates, $\mathbf{u A} / \mathbf{m l}$ & $0.23 \pm 0.06$ & $0.20 \pm 0.09$ & $0.18 \pm 0.09$ & $0.17 \pm 0.05$ \\
\hline Catalase, units by Bach & $0.14 \pm 0.08$ & $0.17 \pm 0.06$ & $0.21 \pm 0.1$ & $0.22 \pm 0.08$ \\
\hline
\end{tabular}

Dihydroquercetin has antioxidant properties and helps to reduce the concentration of following lipid peroxidation products in the blood: malondial dialdehyde from $22.1 \pm 1.1$ at the beginning of the experiment to $16.8 \pm 0.87 \mu \mathrm{mol} / \mathrm{L}$, or by $23.9 \%(\mathrm{p}<0,05)$; diene conjugates from $0.23 \pm 0.06$ to $0.17 \pm 0.05 \mathrm{uA} / \mathrm{ml}$, or $26 \%$; and increase in the activity of catalase from $0.14 \pm 0.08$ to $0.22 \pm 0.08$ units by Bach or by $36 \%$. According to the data obtained, the use of dihydroquercetin positively affects the activity of antioxidant enzymes and lipid peroxidation.

Keeping the service dogs in conditions of intensive working use is associated with the impact of stress factors on the health status of animals. The reduced level of hemoglobin, hematocrit and antioxidant defense of the body can be assumed according to the biochemical parameters of the dog's blood. The prescription of dihydroquercetin in addition to the basic diet of dogs for 30 days can increase the level of anabolic processes, increase the level of body antioxidant protection and thereby prevent the occurrence of animal diseases. Thus, the dihydroquercetin antioxidant optimizes the adaptive capabilities of service dogs and can be used to increase the vitality and longevity of animals.

\section{References}

1. E. Zaplatic, M. Bule, S. Shah, M. Uddin, K. Niaz K, J. Life Sciences, 224, pp. 109-11 (2019)

2. G. Li, X. Shen, Y. Wei, X. Si, J. Wang, J. International Immunopharmacology, 69, pp. $71-78$ (2019)

3. M. Russo, C. Spagnuolo, I. Tedesco, S. Bilotto, G. Russo, Biochem Pharmacol, 83, pp. 6-15 (2012)

4. I. Chen, Y. Tsai, C. Huang, T. Tsai, J. Agric. Food Chem, 58, pp. 546-551(2010)

5. R. Casuso, E. Martínez-Lopez, F. Hita-Contreras, J. Biology of Sport, 31, pp. 63-7 (2014)

6. L. Berger, S. Wein, R. Blank, C. Metges, S. Wolffram, Journal of Dairy Science, 95 (9), pp. 5047-5055 (2012)

7. J. Jung, J. Kang, H. Kim, Nutr. Res. Pract., 6, pp. 301-307 (2012)

8. D'Andrea, G. Quercetin, J. Fitoterapia, 106, pp. 256-71 (2015)

9. A. Burmańczuk, P. Hola, A. Milczak, T. Piech, T. Grabowski, Research in Veterinary Science, 117, pp. 255-259 (2018)

10. Y. Ham, W. Yoon, S. Park, G. Song, J. of Functional Foods, 4 (1), pp. 253-262(2012)

11. M. Oyeyemi, J. of Basic Clinical Physiology and Pharmacology, 23, pp. 39-44 (2012)

12. Y. Yin, W. Li, Y. Sonet, J. Toxicology and Appied Pharmacology, 269 (2), pp. 89-99 (2013)

13. M. Bule, A. Abdurahman, S. Nikfar, M. Abdollahi, M. Amini, Food and Chemical Toxicology, 125, pp. 494-502 (2019) 